\title{
Adaptação do Inventário de Ciúme Romântico (ICR) para a População Portuguesa
}

\author{
JoséMauńdioHaasBueno- UniversidadeFederal dePemanburc, Reife, Pemanburo, Brasil \\ Lucas deFranisco Carvalho- UniveridadeSãoFraniso, Itatiba, SãoPaulo, Brasil \\ Ana Júlia Femandes Mareira - UniversidadedoMinho, Braga, Poturgal \\ Ana Marganida Almida BrandãoCapdo- UniversidadedoMinho, Braga, Paturgal \\ Ângda Marisa Cardoso Femandes- UnivesidadedoMinho, Braca, Portugal \\ Margarida F. Gomes Vascondos- UniveridadedoMinho, Braca, Potugal \\ TiagoAndréMartins Costa - UniversidadedbMinho, Braga, Poturgal \\ Vânia Sofia LeiteMarques- UnivesidadedoMinho, Braca, Poturgl
}

\begin{abstract}
$\overline{\text { Resumo }}$
O objetivo deste estudo foi adaptar um instrumento brasileiro para a avaliação do ciúme romântico no contexto cultural português e avaliar as suas propriedades psicométricas. 0 instrumento adaptado foi respondido por 808 participantes, divididos em duas amostras equivalentes quanto ao número e ao sexo. Análises fatoriais exploratórias revelaram grande similaridade entre as estruturas fatoriais obtidas com as amostras portuguesas e destas com a estrutura encontrada em um estudo prévio com participantes brasileiros. Todos os fatores apresentaram índices de fidedignidade superiores a 0,8. Análises com base na Teoria de Resposta ao Item evidenciaram um bom uso das categorias de resposta empregadas e mostraram que a maioria das escalas é mais discriminativa para características relacionadas com a manifestação patológica do ciúme. Concluiu-se que a escala apresenta validade baseada na estrutura interna e boa fidedignidade, podendo ser recomendada para utilização em investigações.
\end{abstract}

Palavasdhave Ciúme; Avaliação psicológica; Validade; Teoria de Resposta ao Item.

\section{Adaptation of the Romantic Jealousy Inventory for Portuguese People}

\begin{abstract}
The objective of this study was to adapt a Brazilian instrument for romantic jealousy assessment to the Portuguese cultural context, and to evaluate its psychometric properties. The adapted instrument was answered by 808 participants, divided in two equivalent samples regarding number and gender. Simultaneous factor analysis with those samples showed high similarity both with each other and with previous results obtained with a Brazilian sample. All factors showed reliability indices above 0.8. IRT based analysis revealed a good use of the response categories and showed that the majority of the scales are more discriminative for pathologic jealousy manifestation. It was concluded that the scale shows validity based on internal structure and good reliability, and can be recommended for research purposes.
\end{abstract}

Keywards Jealousy; Psychological assessment; V alidity; Item Response Theory.

\section{Adaptación del Inventario de los Celos Románticos (ICR) para la población portuguesa}

\begin{abstract}
Resumen
El objetivo de este estudio fue adaptar un instrumento brasileño para la evaluación de los celos románticos en el contexto cultural portugués y evaluar sus propiedades psicométricas. El instrumento adaptado fue respondido por 808 participantes, divididos en dos muestras equivalentes en cuanto al número y al sexo. Análisis factoriales exploratorias revelaron gran similitud entre las estructuras factoriales obtenidas con las muestras portuguesas y de estas con la estructura encontrada en un estudio preliminar con participantes brasileños. Todos los factores presentaron índices de fiabilidad superiores a 0.8. Análisis basadas en la Teoría de Respuesta al Ítem evidenciaron un buen uso de las categorías de respuesta empleadas y mostraron que la mayoría de las escalas es más discriminativa para características relacionadas con la manifestación patológica de los celos. Se concluye que la escala presenta validez basada en la estructura interna y buena fiabilidad, siendo recomendada para utilización en investigaciones.

Palabras dave Celos; Evaluación psicológica; Validez; Teoría de Respuesta al Ítem.
\end{abstract}

0 ciúme é uma reação natural do ser humano, necessariamente manifestada no contexto de relacionamentos interpessoais, pelo que é considerado por alguns autores como uma emoção social (D eSteno, Bartlett, Braverman \& Salovey, 2002; DeSteno, Valdesolo \& Bartlett, 2006; Harmon-Jones, Peterson \& Harris, 2009; Harris, 2003, 2005). Do ponto de vista evolutivo, o seu aparecimento é associado à necessidade de proteger relacionamentos considerados importantes (Buss, Larsen, Westen \& Semmelroth, 1992), embora este trabalho tenha enfocado apenas 0 ciúme manifestado em contextos amorosos, o ciúme romântico. Por isso, sempre que a palavra ciúme aparecer doravante neste trabalho, os autores querem referir-se ao ciúme romântico.

Nesse contexto, há certa concordância na literatura de que o ciúme é uma experiência desagradável, que provoca reações sentimentais, cognitivas e comportamentais destinadas a proteger 0 relacionamento e/ ou a autoestima, diante da presença de um rival, real ou imaginário, em um relacionamento amoroso (Buunk \& Bringle, 1987; De Silva, 1997; DeSteno \& Salovey, 1996; Pines, 1998; White, 1981). O u seja, do ponto de vista relacional, o ciúme aparece 
quando a exclusividade entre os companheiros è percebida como violada, tenha ela sido de fato ou não (Soble, 1990). Percebe-se, portanto, que são as diferenças disposicionais na interpretação da exclusividade entre companheiros amorosos que levam o ciúme a manifestar-se de forma mais saudável ou mais patológica. No primeiro caso, o ciúme aparece em resposta a evidências concretas de interação companheiro/ a-rival, e as crenças e reações em relação a uma possível traição são mais flexíveis, podendo modificar-se à luz de novas informações (Mullen \& Martin, 1994; Torres, Ramos-Cerqueira \& Dias, 1999). Já na manifestação do ciúme patológico, as crenças ou mesmo delírios de infidelidade são mais frequentes, intensas, rígidas e seguidamente baseadas em evidências infundadas, levando a reações mais extremas, como a agressividade, e mais bizarras, como os comportamentos de investigação (Pfeiffer \& Wong, 1989; Vauhkonen, 1968). Esses comportamentos estão relacionados com a procura por pistas de infidelidade do parceiro, como a contratação de detetives particulares para segui-lo ou a inspeção de mensagens suspeitas em seu telefone celular e e-mail, por exemplo.

Independentemente de o ciúme se manifestar de forma saudável ou patológica, a sensação desagradável decorrente da sua ocorrência tende a desencadear algum tipo de reação. Segundo White (1981), as reações ao ciúme visam à proteção do ego (preservação da autoestima) e/ ou do relacionamento. A agressividade é uma das mais frequentes reações ao ciúme, podendo estar relacionada tanto à proteção do relacionamento, quando dirigida ao rival, como à proteção do ego, quando dirigida ao parceiro (D aly \& Wilson, 1988). Ela pode se manifestar por meio de insultos, ataques físicos dirigidos à pessoa (rival ou parceiro/a) ou aos seus pertences, perseguição, ameaças, entre outras formas, na tentativa de coibir comportamentos de infidelidade pela inflição do medo. Além disso, a mediação da autoestima também é apontada como um fator importante no desencadeamento de reações agressivas. D ois estudos experimentais, realizados por DeSteno e colaboradores (2006) com 46 e 43 universitárias americanas, respectivamente, mostrou que quanto mais uma interação companheiro-rival afetar a autoestima do companheiro rejeitado, maior será a intensidade do ciúme experimentado (estudo 1) e maior a probabilidade de que ocorra uma reação agressiva (estudo 2). No outro extremo, parece legítimo supor que a baixa autoestima aumente a probabilidade de que a causa de uma possível traição seja mais atribuída a deficiências em si mesmo do que no companheiro ou companheira. Essa suposição será testada neste estudo.

Subjacente à ideia de que o ciúme pode se manifestar desde uma forma mais saudável até uma mais patológica, está a noçäo de que há diferenças individuais na manifestação do ciúme. Sendo assim, depreende-se a ideia do ciúme romântico como um traço ou um conjunto de traços latentes, que se manifestam em diferentes intensidades nos indivíduos de uma população.

Foi com base nessa ideia que alguns instrumentos foram propostos para a avaliação do ciúme, tanto como medida unifatorial quanto como medida multifatorial. Entretanto, grande parte desses instrumentos data dos anos 70 e 80 e é de difícil recuperação, uma vez que se trata de comunicações em congressos ou de manuscritos não publicados (Benedictson, 1977; Bryson, 1976, 1977; Hupka \& Bachelor, 1979; Rosmarin, Chambless \& LaPointe, 1979; Rusch \& Hupka, 1977; White, 1980). Por isso, alguns dos instrumentos descritos a seguir apresentam apenas as informações disponíveis na literatura internacional publicadas posteriormente a sua divulgação em contextos mais restritos. Como consequência disso, só podem ser citados de forma secundária.

Como exemplos de escalas unidimensionais podem ser citados a Dispositional Jealausy Scale(Nadler \& Dotan, 1992), composta por 14 itens $(a=0,85)$, e a Interpesscal Jealusy Scale (Mathes, Phillips, Skowran \& Dick, 1982, citados por De Silva, 1997), composta por 27 itens. Embora o coeficiente de fidedignidade dessa última não seja referido, a autora sugere que a escala seja consistente.

A complexidade da manifestação do ciúme levou muitos autores a sugerirem que sua avaliação deveria ser multifatorial (Mathes, 1992; Pfeiffer \& Wong, 1989; Pines, 1992). Em linha com essa proposição está 0 Ramantic Jealausy Questionmaire (RJQ ; Pines \& Aronson, 1983, citados por Demirtas \& Dönmez, 2006), que avalia cinco domínios relacionados ao ciúme: intensidade, reações (física, emocional, cognitiva), formas de enfrentamento e causas e efeitos. 0 número de itens e 0 coeficiente de fidedignidade de cada escala não foram informados.

Outra importante escala para avaliação multifatorial do ciúme é a Multidimenional Jęlausy Scale (MJS), que foi desenhada para captar as manifestações cognitivas, emocionais e comportamentais do ciúme romântico. Três estudos, realizados com 178, 123 e 76 sujeitos, respectivamente, relativamente equilibrados quanto ao sexo e recrutados em ambiente universitário, atestaram a estabilidade da estrutura fatorial dessa escala, sempre com índices de consistência interna superiores a 0,80 (Pfeiffer \& Wong, 1989).

Não se tem notícia de um instrumento semelhante para a população portuguesa, mas foram encontrados três instrumentos recentes em português, 
para população brasileira (Bueno \& Carvalho, no prelo; Carvalho, Bueno \& Kebleris, 2008; Ramos, Y azawa \& Salazar, 1994). Ramos e colaboradores (1994) obtiveram um instrumento com 58 itens, que foram agrupados em três fatores: nãoamaça $(a=0,87)$, que se refere ao caráter não-perturbável da relação amorosa pela interferência de uma terceira pessoa; exdusão $(a=0,85)$, que descreve 0 enfraquecimento da relação do casal pela interferência de um rival; e intefêênia $(a=0,77)$, relativo a reações agressivas provocadas pela interferência de um rival. Embora tenha apresentado boas propriedades psicométricas, Bueno e Carvalho (no prelo) referem que esse instrumento apresenta fundamentação mais empírica do que teórica, não contemplando a avaliação de certos aspectos frequentemente associados ao ciúme, como os comportamentos de investigação, a baixa autoestima e os pensamentos obsessivos de traição.

Por isso, em dois trabalhos (Bueno \& Carvalho, no prelo; Carvalho \& cols., 2008) os autores desenvolveram o Inventário de Ciúme Romântico (ICR), cuja versão final ficou com 30 itens agrupados em seis fatores, capazes de explicar $60 \%$ da variância total. $\mathrm{O}$ primeiro fator (Não-Contacto com o Parceiro, $a=0,90$ ) reuniu itens que descrevem crenças ou pensamentos de infidelidade quando um contacto com o parceiro não é conseguido, ou perante a perda de controle sobre a localização do parceiro. O segundo fator (Contacto Parceiro-Rival, $a=0,83$ ) agrupou itens relacionados a reações emocionais negativas ante a situações de violação da exclusividade. $O$ terceiro e 0 quarto fatores referem-se a reações agressivas dirigidas ao companheiro/ a (Agressão ao Parceiro, $a=0,81$ ) ou ao rival (Agressão ao Rival, $a=0,66)$, respectivamente. 0 quinto fator (Autoestima, $a=0,67$ ) refere-se a reações em que o companheiro rejeitado tenha se culpado pela possibilidade de infidelidade do parceiro. 0 último fator (F6 - Investigação, $a=0,55$ ) reuniu itens que se referem a comportamentos de investigação relacionados à (in)fidelidade do parceiro (Bueno \& Carvalho, no prelo).

Observa-se, portanto, que foram obtidos os fatores esperados e compatíveis com a literatura. Entretanto, os três últimos fatores apresentaram índices de fidedignidade abaixo do esperado pelos autores $(0,70)$, fato que foi atribuído ao baixo número de itens que esses fatores apresentaram (Bueno \& Carvalho, no prelo).

Dada a carência de um instrumento para a avaliação do ciúme romântico na população portuguesa, o evidente impacto na vida pessoal e social que esse fenômeno pode causar e a necessidade de um instrumento para realização de investigações nessa área, o objetivo principal deste trabalho foi adaptar 0
Inventário de Ciúme Romântico para 0 português europeu e investigar as suas propriedades psicométricas numa amostra da população portuguesa. Especificamente, pretendeu-se verificar se a estrutura fatorial encontrada no estudo brasileiro se reproduziria em duas amostras de participantes portugueses; se as amostras de participantes portugueses produziriam estruturas fatoriais semelhantes entre si e se a revisão e acréscimo de itens, especialmente em relação aos três últimos fatores do instrumento brasileiro, resultaria numa melhoria dos índices de fidedignidade, caso os fatores se reproduzissem. Além disso, empregou-se 0 Modelo de Rasch da Teoria de Resposta ao Item para verificar 0 ajustamento dos itens a esse modelo, analisar as probabilidades de ocorrência das categorias de resposta empregadas no instrumento (escala do tipo Liket, de 1 a 4) e a relação entre a intensidade de ciúme avaliada pelos itens que compõem o instrumento e a apresentada pelos participantes da investigação.

\section{Partiapantes}

\section{Método}

A coleta de dados foi aberta à participação de alunos de uma universidade pública do norte de Portugal, contatados por correio eletrônico e incentivados a estender 0 convite a outros possíveis interessados. A amostra total foi constituída por 808 sujeitos, distribuídos equitativamente quanto ao sexo. Entre os que informaram idade $(\mathrm{N}=800)$, a média foi de 23,2 e o desvio padrão de 4,4 anos, tendo variado de 17 a 41 anos. Para realização da análise fatorial o grupo total foi dividido em dois subgrupos de 404 participantes, também equitativos quanto ao sexo. Uma análise univariada de variância mostrou não haver diferenças significativas de idade entre esses grupos $(F=1,840 ; p=0,174)$, nem entre os sexos $(F=3,139$; $\mathrm{p}=0,077)$, nem tampouco interação entre grupo e sexo $(\mathrm{F}=0,145 ; \mathrm{p}=0,704)$.

\section{Instrumentos}

Inicialmente, os 30 itens do Inventário de Ciúme Romântico - ICR (Bueno \& Carvalho, no prelo) foram adaptados do português brasileiro para 0 europeu e para a cultura portuguesa. Nesse processo, algumas palavras foram alteradas em relação à grafia, recebendo 0 " $c$ " mudo, como contado facto e djeto por exemplo, ou outras pequenas modificações, como rejstoao invés de registro. Outras palavras ou expressões foram substituídas por sinônimos mais usuais no português europeu, como companhero/a, preferível a parceiro/a, tdemód, mais usual do que celular e "faço uma cma" ao invés de "armo barraco". Houve ainda alterações sintáticas para evitar o uso de gerúndio (como estar a dhar, au estar a trair ao invés de olhando e traindo, respectivamente) ou adequar à maneira portuguesa 0 
uso de pronomes pessoais (como, panso que ae a me pode etar a trair ao invés de penso que ele/ a pode estar me traindo) e reflexivos (como, a convesar com alguém comquemjá se tenha rdacionadoao invés de com quem já tenha se relacionado).

Além disso, seguindo sugestão dos próprios autores da versão brasileira do instrumento, foram desenvolvidas mais frases para todas as subescalas, na tentativa de melhorar os índices de fidedignidade e de representar melhor as diferentes facetas do constructo estudado. Esse procedimento foi facilitado pela composição da equipe de investigação, que contou com dois participantes brasileiros, sendo um doutor e outro doutorando, ambos em avaliação psicológica; e seis portugueses, sendo uma mestranda em Psicologia e cinco estudantes de graduação. Assim, no instrumento final houve um aumento de seis para nove nos itens que se referiam a reações emocionais ligadas a violações de exclusividade, que descreviam cenas de contato entre parceiro e rival; de sete para onze itens que se referiam a ocorrência de pensamentos de infidelidade em situações em que um contacto com o/ a companheiro/ a não era conseguido; de quatro para dez itens que descreviam reações cognitivas de culpa em relação a si mesmo e de baixa autoestima ante a possibilidade de infidelidade do/a companheiro/ a; de seis para dez itens que se referiam a reações agressivas dirigidas ao companheiro; de quatro para seis itens sobre reações agressivas dirigidas ao rival e de três para nove itens referentes a comportamentos de investigação em decorrência da desconfiança de infidelidade do/ a companheiro/ a.

Portanto, a versão final do instrumento adaptado ficou com cinquenta e cinco itens para serem respondidos por meio de uma escala Liket de quatro pontos: 1- nada ou muito pouco característico até 4 extremamente ou totalmente característico. Finalmente, essa versão foi formatada na ferramenta G oogleD ocs, para que pudesse ser acedida pelos participantes via internet.

\section{Procedimentos}

Os participantes foram contatados por correio eletrônico e informados acerca dos objetivos da investigação, do caráter voluntário da participação e da confidencialidade quanto ao tratamento dos dados. Além disso, como retribuição à sua participação, após a coleta e análise dos dados, os participantes receberam uma mensagem eletrônica com seus resultados comentados e um contato para o caso de desejarem outras informações, o que não foi utilizado por nenhum participante. As suas respostas foram automaticamente guardadas num ficheiro Excel, cujos dados toram transportados para a versão 17.0 do Statistical Padkagefor Social Saieneses(SPSS 17.0).

Considerando 0 objetivo de verificar a reprodutibilidade da estrutura fatorial, tanto em relação ao estudo brasileiro quanto entre participantes portugueses (amostras independentes), o banco de dados foi dividido em dois grupos equitativos quanto ao número e ao sexo dos participantes. Cada grupo foi analisado independentemente, mas segundo os mesmos procedimentos: verificação da fatarabilidadeda matriz de correlações, definição do número de fatores a serem retidos e a extração dos fatores propriamente ditos. Os coeficientes alfa de Cronbach foram computados para verificação da consistência interna dos fatores extraídos. Finalmente, empregou-se 0 modelo de Rasch da Teoria de Resposta ao Item para analisar a eficiência das categorias de resposta empregadas no instrumento (1 a 4) e a relação entre a intensidade do ciúme avaliada pelos itens e a apresentada pelos participantes da investigação.

\section{Resultados e discussão}

0 primeiro passo da análise de dados foi a verificação das condições para a realização da análise fatorial exploratória por meio: (1) da inspeção da matriz de correlações; (2) do Teste de Esfericidade de Bartlett e (3) do cálculo do índice de adequação da amostra de Kaiser-Meyer-Olkin (KMO). A inspeção da matriz de correlações mostrou que a média das correlações no grupo 1 foi de 0,268 e a do grupo 2 foi de 0,270. 0 Teste de Esfericidade de Bartlett mostrou que ambas as matrizes de correlações eram significativamente diferentes de uma matriz identidade, com $\chi^{2}(\mathrm{gl}=1485)=12005,3$ e $\mathrm{p}=0,001$ para 0 grupo 1 e $\chi^{2}(\mathrm{gl}=1485)=12611,9$ e $\mathrm{p}=0,001$ para 0 grupo 2. Por fim, 0 KMO foi de 0,932 e 0,933 para os grupos 1 e 2, respectivamente. Segundo critérios sumariados por Tabachnick e Fidell (1989), esse conjunto de dados indica que há correlações suficientes entre as variáveis para a realização da análise fatorial em ambos os grupos, o que permitiu a passagem para 0 segundo passo na investigação da estrutura fatorial: a definição do número de fatores a serem retidos.

Para isso, empregou-se o método da análise paralela, recomendado e descrito por Reise, Waller e Comrey (2000). Utilizou-se o satware RanEigen (Enzmann, 1997) para a obtenção dos dados aleatórios e seus respectivos eigenvalues cuja comparação com os dados experimentais resultou na retenção dos primeiros cinco fatores. Com base nisso, impôs-se a extração de cinco fatores pelo método dos eixos principais e rotação dbimin devido à expectativa da ocorrência de correlações significativas entre os fatores. 
Com vistas à obtenção da estrutura mais simples possível, procedeu-se a uma inspeção das cargas fatoriais dos 55 itens que compunham a escala inicial, eliminando-se os itens que apresentassem: (1) carga fatorial inferior a 0,32 em todos os fatores (Tabachnick \& Fidell, 1989); (2) cargas fatoriais superiores a 0,32 em mais de um fator, eliminando-se 0 item da análise ou apenas do fator com menor carga, conforme a diferença entre as cargas fosse inferior ou superior a 0,1, respectivamente; (3) comprometimento da consistência interna do fator correspondente; (4) incompatibilidade entre o seu conteúdo e os dos demais no mesmo fator. Nas análises de ambos os grupos foram eliminados 11 itens, sendo 10 comuns (4, $5,6,11,16,28,44,50,51$ e 53) e dois discrepantes: 0 item 12 foi eliminado apenas da análise do grupo 1 e 0 34 apenas da análise no grupo 2. Os itens eliminados eram esperados nos fatores NãoContadto com 0 Companhird a (44 e 53), Compatamento Agressivo (5, 6, 11, 12 e 51) e de Inmesticação $(4,16,28,34$ e 50). Uma análise da estruturação semântica não revelou qualquer padrão que pudesse explicar o mau funcionamento psicométrico desses itens, que foram, entretanto, eliminados em razão das evidências empíricas. Dessa forma, obtiveram-se as soluções fatoriais finais apresentadas na Tabela 1, onde as cargas referentes ao grupo 2 estão entre parênteses.

A observação da Tabela 1 mostra que as estruturas fatoriais obtidas com ambas as amostras de participantes foram praticamente idênticas entre si e muito semelhantes à estrutura encontrada com a população brasileira (Bueno \& Carvalho, no prelo). Após a rotação oblíqua dos eixos, as soluções fatoriais explicaram 48,7\% e 48,6\% da variância total para 0 primeiro e 0 segundo grupo de participantes, respectivamente. 0 primeiro fator apresentou eigenvalues de 12,4 e 13,0, explicando 28,3 e 29,5\% da variância total por meio dos mesmos 12 itens para os grupos $1 \mathrm{e}$ 2 , respectivamente. Apesar disso, na análise do grupo 2, 0 item 39 (Quando conbino algo com od a mal minha companherd a edel a seatrasa, penso quefa porqueeterecom atra/ o milhed homem) também apresentou carga no quarto fator $(0,350)$, ainda que bastante inferior à apresentada no primeiro $(0,568)$. A incompatibilidade entre 0 conteúdo desse item e 0 dos demais do fator 4 sugere a sua permanência e cotação apenas no primeiro fator. No conjunto, os itens do primeiro fator descrevem reações cognitivas (penso, desconfio, concluo) relacionadas com a infidelidade em situações em que não se consegue entrar em contacto com o(a) companheiro(a) (e.g. item 27: "Quando eu não consigo entrar em contacto com o/ a meu/minha companheiro/a, penso que ele/a me pode estar a trair."), sendo, assim, claramente compatível com 0 primeiro fator da versão brasileira, denominado Não ContadocomoParciro(Bueno \& Carvalho, no prelo).

0 segundo fator reuniu os mesmos 11 itens com as amostras dos grupos 1 e 2, explicando 7,9 e 7,1\% da variância total, com égenvalues iguais a 3,5 e 3,1, respectivamente. Esse conjunto de itens descreve reações emocionais negativas (por exemplo, "é desconfortável", "é desagradável", "sinto-me mal", "perturba-me", "incomoda-me") perante a constatação de algum tipo de interação companheiro/a-rival (por exemplo, item 42, "Sinto-me desconfortável quando o/ a meu/minha companheiro/a chama por nomes carinhosos outra/ o mulher/ homem"). Dessa forma, 0 segundo fator da versão portuguesa é claramente compatível com o segundo fator da versão brasileira, tendo sido designado de Contado ParceroRival (Bueno \& Carvalho, no prelo).

Os 10 itens que se agruparam no terceiro fator explicam 5,2\% da variância total, com eignvalueigual a 2,3 em ambos os grupos analisados. Referem-se a reações de culpa em relação a si mesmo diante da possibilidade de interação companheiro/a-rival (e.g. item 08 "Provavelmente eu serei a/ o responsável se o/ a meu/ minha companheiro/ a se interessar por outra pessoa") e/ ou baixa autoestima em relação ao companheiro/a (e.g. item 14, "Acho que não sou bom/boa 0 suficiente para a/ o minha/meu companheira/ o no nosso relacionamento"). Além dos itens nesse conjunto serem idênticos entre si neste estudo, também são claramente compatíveis com 0 quinto fator da versão brasileira, denominado de (baixa) Autoestima (Bueno \& Carvalho, no prelo).

0 quarto fator reuniu 8 e 9 itens, capazes de explicar 4,3 e 4,0\% da variância total, com égenvalues iguais a 1,9 e 1,7 para os grupos 1 e 2, respectivamente. Nesse caso, todos os itens são idênticos entre os dois grupos, com exceção do item 12 ("Discuto com a pessoa que acho que se está a relacionar com o/ a meu/minha companheiro/a"), que foi excluído das análises do grupo 1 por apresentar carga fatorial inferior a 0,32 em todos os fatores, mas que apresentou carga de 0,435 no quarto fator, com os dados do grupo 2. De qualquer forma, esse item é compatível com os demais que descrevem reações de confronto tanto ao companheiro (e.g. item 46, "Agrido fisicamente o/ a $\mathrm{meu} /$ minha companheiro/ a por achar que este me está a ser infiel.") quanto ao rival (e.g. item 30, "Persigo a pessoa com quem eu acho que o/a meu/minha companheiro/ a está a ter um caso"), aquando da suspeita de infidelidade. Esse fator reproduziu-se de forma muito semelhante entre as amostras analisadas neste estudo, mas reuniu comportamentos agressivos dirigidos ao companheiro/a e ao rival, enquanto a versão brasileira apresentava fatores distintos em 
função do alvo do comportamento agressivo (Bueno \& Carvalho, no prelo). Embora não seja possível compreender o porquê dessa diferença, ela não compromete a ideia de que a estrutura se reproduziu, dada a relação entre comportamentos agressivo e 0 ciúme mencionada em estudos anteriores (Bueno \& Carvalho, no prelo; Carvalho \& cols., 2008; DeSteno \& cols., 2006; Ramos \& cols., 1994).

Tabela 1. Cargas fatoriais e coeficientes alfa de ambos os grupos ${ }^{1}$

\begin{tabular}{|c|c|c|c|c|c|}
\hline Item & Fator 1 & Fator 2 & Fator 3 & Fator 4 & Fator 5 \\
\hline 03 & $0,716(0,711)$ & & & & \\
\hline 09 & $0,480(0,434)$ & & & & \\
\hline 15 & $0,738(0,747)$ & & & & \\
\hline 21 & $0,776(0,760)$ & & & & \\
\hline 27 & $0,838 \quad(0,819)$ & & & & \\
\hline 33 & $0,663(0,563)$ & & & & \\
\hline 39 & $0,544(0,554)$ & & & $(0,350)$ & \\
\hline 49 & $0,723(0,660)$ & & & & \\
\hline 52 & $0,573(0,514)$ & & & & \\
\hline 55 & $0,726(0,692)$ & & & & \\
\hline 01 & & $-0,535 \quad(-0,484)$ & & & \\
\hline 07 & & $-0,628(-0,717)$ & & & \\
\hline 13 & & $-0,791 \quad(-0,772)$ & & & \\
\hline 17 & & $-0,387 \quad(-0,369)$ & & & \\
\hline 18 & & $-0,474 \quad(-0,549)$ & & & \\
\hline 19 & & $-0,800 \quad(-0,773)$ & & & \\
\hline 25 & & $-0,506(-0,658)$ & & & \\
\hline 31 & & $-0,770(-0,720)$ & & & \\
\hline 37 & & $-0,561 \quad(-0,476)$ & & & \\
\hline 42 & & $-0,811 \quad(-0,806)$ & & & \\
\hline 47 & & $-0,650(-0,651)$ & & & \\
\hline 02 & & & $0,555(0,448)$ & & \\
\hline 08 & & & $0,625(0,584)$ & & \\
\hline 14 & & & $0,570(0,669)$ & & \\
\hline 20 & & & $0,480(0,554)$ & & \\
\hline 26 & & & $0,591 \quad(0,638)$ & & \\
\hline 32 & & & $0,615(0,713)$ & & \\
\hline 38 & & & $0,412(0,504)$ & & \\
\hline 43 & & & $0,458(0,520)$ & & \\
\hline 48 & & & $0,617 \quad(0,629)$ & & \\
\hline 54 & & & $0,590(0,517)$ & & \\
\hline 12 & & & & Excl. $(0,435)$ & \\
\hline 23 & & & & $0,493(0,508)$ & \\
\hline 24 & & & & $0,477(0,338)$ & \\
\hline 29 & & & & $0,710(0,651)$ & \\
\hline 30 & & & & $0,592(0,584)$ & \\
\hline 35 & & & & $0,517 \quad(0,477)$ & \\
\hline 36 & & & & $0,519(0,446)$ & \\
\hline 41 & & & & $0,706(0,547)$ & \\
\hline 46 & & & & $0,753(0,694)$ & \\
\hline 10 & & & & & $0,670(0,594)$ \\
\hline 22 & & & & & $0,767 \quad(0,702)$ \\
\hline 34 & & & & & 0,433 (Excl.) \\
\hline 40 & & & & & $0,610(0,483)$ \\
\hline 45 & & & & & $0,714(0,627)$ \\
\hline$\alpha$ & $0,914(0,908)$ & $0,903(0,904)$ & $0,832(0,836)$ & $0,847(0,828)$ & $0,833(0,857)$ \\
\hline
\end{tabular}

1 "A tabela com os itens completos pode ser obtida por meio de solicitação ao autor principal via e-mail." 
Finalmente, o quinto fator ficou com 5 e 4 itens capazes de explicar 3,0 e 2,8\% da variância total, correspondente a égenvalues iguais a 1,3 e 1,2 para os dados dos grupos 1 e 2, respectivamente. Uma pequena modificação ocorreu devido ao item 34 ("Quando ouço o/ a meu/minha companheiro/a a falar com alguém, tento ouvir a conversa"), que apresentou carga de 0,433 no quinto fator para os dados do grupo 1, mas que foi excluído do quinto e do segundo fator, com os dados do grupo 2, por apresentar cargas com diferença inferior a 0,1. De qualquer forma, esses itens retratam comportamentos de investigação e/ ou espreita decorrentes da desconfiança sobre a fidelidade do/a companheiro/ a (por exemplo, item 22, "Quando o/ a $\mathrm{meu} / \mathrm{minh}$ companheiro/a se esquece do telemóvel, vou ver a caixa de mensagens e o registo de chamadas para saber se está a ter um caso"). Esse fator é compatível com a sexta dimensão da versão brasileira denominada Invesigaç̃a(Bueno \& Carvalho, no prelo).

Para verificar a possibilidade de reagrupamento dos cinco fatores primários, foi realizada uma análise fatorial de segunda ordem. Corroborando os resultados obtidos por Bueno e Carvalho (no prelo), obteve-se solução unifatorial com ambas as amostras. Os égenalues variaram de 0,506 e 0,483 (Autoestima) a 0,774 e 0,835 (Não-contato com o/ a companheiro/ a) com as amostras 1 e 2 , respectivamente. Além de confirmar a unifatorialidade do construto, esse dado permite 0 cômputo de uma pontuação baseada na média dos fatores primários, relacionada com a manifestação geral do ciúme.

Para determinação da consistência interna das escalas, foram calculados os coeficientes alfa de Cronbach, cujos resultados podem ser observados nas Tabelas 1 e 2. Os valores foram sempre superiores a 0,8 , tal como desejado pelos padrões internacionais, que exigem, no mínimo, 0,7. Uma boa consistência interna permite afirmar que as diferenças individuais encontradas podem ser atribuídas mais aos efeitos do constructo mensurado do que a erros de medida (Urbina, 2007).

Na sequência das análises, empregou-se o Modelo de Rasch da Teoria de Resposta ao Item (TRI), com 0 objetivo de: (a) verificar o ajustamento dos itens ao modelo da TRI; (b) analisar a Escala Global quanto às probabilidades de ocorrência das categorias de resposta e (c) quanto à relação entre a intensidade de ciúme avaliada pelos itens do instrumento e a apresentada pelos participantes da investigação.

Para verificar o ajustamento dos itens ao modelo da TRI, foram empregados os índices de infit e atfit, cujos valores entre 0,5 e 1,5 são indicativos de um bom ajustamento (Linacre, 2002). A média de infit foi de $1,05(\mathrm{DP}=0,19)$ e a de aufit foi de 0,97 $(\mathrm{DP}=0,30)$.
Apenas três itens apresentaram autfits elevados (2, 14 e $20)$, todos relacionados com 0 terceiro fator, que descreve reações de baixa autoestima. Entretanto, os valores situaram-se entre 1,5 e 2,0, faixa considerada como improdutiva, mas não degradante para a medida (Linacre, 2002), pelo que se considerou o ajustamento como adequado para o prosseguimento das análises.

Para investigar a manutenção do paralelismo entre as categorias de resposta empregadas no instrumento e a intensidade de manifestação do constructo (tła), recorreu-se à análise das probabilidades de ocorrência das categorias de resposta. Os resultados dessa análise indicaram que a categoria "1" é a mais provável até um nível de intensidade do ciúme (tta) igual a -0,92. Entre esse valor e -0,10, a categoria " 2 " passa a ser a mais provável, seguida pela categoria " 3 ", para valores de tta entre -0,10 e 1,02, e, finalmente, pela categoria " 4 " para valores de tta acima de 1,02. De forma semelhante, os valores médios de tta foram de -2,64 para a categoria "1", -1,15 para a categoria "2", -0,36 para a categoria "3" e 0,36 para a categoria "4". Portanto, conforme esperado, tanto os valores médios de tda quanto os valores de tła correspondentes à transição de uma categoria para outra form aumentando consoante 0 aumento da categoria de resposta em si, mostrando que há paralelismo entre as categorias de resposta empregadas no instrumento e a intensidade de manifestação do constructo, e que todas as categorias de resposta foram úteis para a discriminação dos sujeitos numa faixa significativa da amplitude total de manifestação do constructo.

Finalmente, foram computadas as estatísticas descritivas de cada fator pelo modelo de Rasch, cujos dados são apresentados na Tabela 2. Nota-se que a média tende a ficar abaixo do ponto central da distribuição (zero), sugerindo que a escala é sensível à manifestação patológica do ciúme romântico. Uma exceção foi observada no segundo fator (contacto companheiro-rival), em que a média é claramente mais elevada em relação aos outros. Isso mostra que os participantes tenderam a atribuir valores mais altos aos itens desse fator do que ao de outros, sugerindo que 0 segundo fator seja um indicador de reações mais frequentes, enquanto os demais indiquem reações menos frequentes na população.

Dessa forma, os fatores obtidos podem ser interpretados como reações desagradáveis e/ ou danosas em decorrência de uma interação entre o/ a companheiro/ a e um possível nival. Entretanto, o segundo fator diferencia-se dos demais por descrever reações amenas (é desagradável, não me sinto bem, entre outras) a uma interação companheiro/ a-rival que é efetivamente observada pela parte rejeitada e normalmente interpretada como violação da 
exclusividade (Soble, 1990). Essas são características da manifestação mais comumente observada e nãopatológica do ciúme romântico (Pfeiffer \& Wong, 1989; Vauhkonen, 1968). Todos os demais fatores, por sua vez, descrevem reações mais intensas, disruptivas e/ ou danosas a, pelo menos, uma das partes envolvidas, pelo que podem ser considerados como indicadores de manifestações mais patológicas do ciúme romântico (Pfeiffer \& Wong, 1989).

Tabela 2. Estatísticas descritivas dos fatores do ICR

\begin{tabular}{lccccc}
\hline Fatores & $\mathrm{N}$ & Mínim0 & Máximo & Média & D esvio padrão \\
\hline Fator 1 & 808 & $-5,83$ & 5,62 & $-3,49$ & 1,95 \\
Fator 2 & 808 & $-5,30$ & 5,35 & $-0,62$ & 1,61 \\
Fator 3 & 808 & $-4,94$ & 5,05 & $-2,14$ & 1,48 \\
Fator 4 & 808 & $-4,99$ & 4,98 & $-3,99$ & 1,35 \\
Fator 5 & 808 & $-4,71$ & 4,72 & $-2,60$ & 1,95 \\
Fator G eral & 808 & $-6,51$ & 6,72 & $-1,92$ & 1,12 \\
\hline
\end{tabular}

De qualquer forma, guardadas as especificidades de cada fator, pontuações elevadas sugerem maior propensão ao sofrimento psicológico e ao comprometimento da qualidade do relacionamento em decorrência do ciúme. Por sua vez, pontuações baixas podem ter várias interpretações, como 0 relacionamento já não ser importante para o/a companheiro/a "rejeitado/a", o/a companheiro/a rejeitado/ a ser algo ingênuo/ a e não perceber a ameaça ao relacionamento, a interação entre o/ a companheiro/ a e uma terceira pessoa causar prazer no/ a companheiro/ a "rejeitado/ a" ou o companheiro rejeitado não apresentar 0 mínimo de energia necessária para a defesa do seu relacionamento e/ ou da sua autoestima. Embora essas interpretações sejam prováveis, elas ainda são meramente especulativas neste momento, mas podem (e devem) constituir-se em objeto de futuras investigações especialmente desenhadas para essa finalidade.

\section{Considerações finais}

Este estudo pretendeu adaptar o Inventário de Ciúme Romântico para o contexto cultural português e investigar suas propriedades psicométricas. Para isso, empregou recursos da Teoria Clássica dos Testes ao lado de recursos da moderna Teoria de Resposta ao Item. Os resultados apontaram para a robustez do constructo estudado, dada a grande semelhança tanto entre as estruturas obtidas com a amostra brasileira e portuguesa quanto entre duas amostras de participantes portugueses.

Além disso, os resultados também indicam a robustez do próprio instrumento, que mostrou ser eficaz para avaliação de cinco dimensões do ciúme romântico compatíveis com proposições teóricas e com bons índices psicométricos. Por isso, embora ainda sejam necessários estudos com amostras mais amplas e representativas de toda a extensão do territónio português, o presente instrumento pode ser recomendado para 0 uso em investigação, como uma medida satisfatória de diferentes aspectos relacionados ao ciúme. E, nesse sentido, abre-se, por um lado, a possibilidade de utilização do instrumento em psicologia clínica, aonde a manifestação do ciúme é relatada como um dos temas mais frequentes tanto em terapias individuais (Costa, 2005) quanto de casal (Leite, 2000). E, por outro, contribui para a realização de mais pesquisas sobre 0 fenômeno do ciúme, tão frequente e impactante socialmente, especialmente no que se refere à violência doméstica (ver Kingham \& Gordon, 2004), e tão pouco estudado cientificamente.

\section{Referências}

Benedictson, C. (1977). The dadopmet of a scale for the assesment of jeglasy. Trabalho apresentado no encontro de Southeastern Psychological Association.

Bryson, J. B. (1976). The nature of sexual jeflasy: an explaratay sudy. Trabalho apresentado no encontro de American Psychological Association, Washington, D.C.

Bryson, J. B. (1977). Situational deteminants of the expression of jexlausy. Trabalho apresentado no encontro de American Psychological Association, San Francisco.

Bueno, J. M. H., \& Carvalho, L. F. (no prelo). Construção de um inventário para avaliação do ciúme romântico. Psicdogia: RelexãoeCńtica

Buss, D. M., Larsen, R., Westen, D ., \& Semmelroth, J. (1992). Sex differences in jealousy: evolution, physiology, and psychology. Psychological Saience, 3, 251-255.

Buunk, B., \& Bringle, R. G. (1987). Jealousy in love relationships. Em D. Perlman \& S. Duck (Ed.), 
Intimate rdationships dexdqpment, dynamics and deteioration(pp. 123-147). Beverly Hills, CA: Sage.

Carvalho, L. F., Bueno, J. M. H., \& Kebleris, F. (2008). Estudos psicométricos preliminares do Inventário de Ciúme Romântico - ICR. AvaliaçãoPsicdógia, 7, 335-346.

Costa, N. (2005). Contribuições da psicologia evolutiva e da analíse do comportamento acerca do ciúme. Revista Brasileira deTeapia Compatametal Cogritiva, 7, 05-14

Daly, M., \& Wilson, M. (1988). Evolutionary social psychology and family homicide. Saience, 242, 519524.

De Silva, P. (1997). Jealousy in couple relationships: nature, assessment and therapy. Behaviar Reserch and Therapy, 35, 973-985.

Demirtas, H. A., \& Dönmez, A. (2006). Jealousy in close relationships: personal, relational and situational variables. TurkishJaumal of Psychiatry, 17, $1-11$.

DeSteno, D. A., \& Salovey, P. (1996). Evolutionary origins of sex differences in jealousy? Questioning the "fitness" of the model. Psyddogical Saiene, 7, 367-372.

DeSteno, D., Bartlett, M. Y., Braverman, J., \& Salovey, P. (2002). Sex differences in jealousy: evolutionary mechanism or artifact of measurement? Joumal of Pessonalityand Social Psychdogy, 83, 1103-1116.

DeSteno, D., Valdesolo, P., \& Bartlett, M. Y. (2006). Jealousy and the threatened self: getting to the heart of the green-eyed monster. Jaumal of Pessonalityand Social Psychdogy, 91, 626-641.

Enzmann, D. (1997). RanEigen: a program to determine the parallel analysis criterion for the number of principal components. Applied Psychdogical Measurement, 21, 232.

Harmon-Jones, E., Peterson, C. K., \& Harris, C. R. (2009). Jealousy: novel methods and neural correlates. Emotion, 9, 113-117.

Harris, C. R. (2003). A review of sex differences in sexual jealousy, including self-report data, psychophysiological responses, interpersonal violence, and morbid jealousy. Pessanality and Social PsychdogyReview 2, 102-128.

Harris, C. R. (2005). Male and female jealousy, still more similar than different: replay to sagarin. Pesonalityand Social Psychdogy Review 1, 76-86.
Hupka, R. B., \& Bachelor, B. (1979). Validation of a scale tomasureromanticjealausy. Trabalho apresentado no encontro de Western Psychological Association, San Diego.

Kingham, M., \& Gordon, H. (2004). Aspects of morbid jealousy. Advances in Psychiatric Treatment, 10, 207-215.

Leite, S. M. C. S. (2000). Ciúme e inveja: a visão comportamental. Em R. C. Wielenska (Ed.), Sdbre compatamento e cogniçãa questionando e ampliandb a teeria eas intervencós dínicas e ematros contextos (vol. 6, pp. 74-77). Santo André: ESETec Editores Associados.

Linacre, J. M. (2002). What do Infit and Outfit, meansquare and standardized mean? Rasch Measurement Transadions, 16, 878.

Mathes, E. W. (1992). Jealousy: the psydological data Lanham: University Press of America.

Mullen, P. E., \& Martin, J. L. (1994). Jealousy: a community study. British Jaumal of Psychiatry, 164, $35-43$.

Nadler, A., \& D otan, I. (1992). Commitment and rival attractiveness: their effects on male and female reactions to jealousy-arousing situations. Sex Rdes 26, 283-310.

Pfeiffer, S. M., \& Wong, P. T. P. (1989). Multidimensional jealousy. Jaumal of Social and Pessonal Rdationships, 6, 181-196.

Pines, A. M. (1992). Romantic jealousy: five perspectives and an integrative approach. Psychotherapy, 29, 675-683.

Pines, A. M. (1998). Ramantic jealasy: causes symptoms ares Nova Iorque: Routledge.

Ramos, A. L. M., Yazawa, S. A. K., \& Salazar, A. F. (1994). D esenvolvimento de uma escala de ciúme romântico. Psicdoga: Teeria ePesquisa, 10, 439-451.

Reise, S. P., Waller, N. G., \& Comrey, A. L. (2000). Fator analysis and scale revision. Psydhdogical Assesment, 12, 287-297.

Rosmarin, D. M., Chambless, D. L., \& LaPointe, K. (1979). Thesurvey of interpessomal reacions an inventary for the masurement of jeglausy. Manuscrito não publicado, University of G eorgia.

Rusch, P. A., \& Hupka, R. B. (1977). Deddpmett and validation of a scale to masure romantic jealousy. Trabalho apresentado no encontro annual de Western Psychological Association, Seattle. 
soble, A. (lyyu). Ine structure of love $\mathrm{Y}$ ale: $\mathrm{Y}$ ale University Press.

Tabachnick, B. G., \& Fidell, L. S. (1989). Using multivaniate statistics ( $2^{\mathrm{a}}$ ed.). Cambridge: Harper \& Row.

Torres, A. R., Ramos-Cerqueira, A. T. A., \& Dias, R. S. (1999). O ciúme enquanto sintoma do transtorno obsessivo-compulsivo. Reista Brasilera de Psiquiatria, 21(3), 165-173.

Urbina S. (2007). Fundamentos da testagem psicdóġa Porto Alegre: Artmed.
V aunkonen, K. (Iybz). Un the pathogenesis of morbid jealousy. Ada Psychiatrica Scandinavica Supplementum, 202, 2-261.

White, G. L. (1980). Validation of three jealausy scales Manuscrito não-publicado, University of Maryland, College Partk.

White, G. L. (1981). A model of romantic jealousy. MotivationandEmotion, 5(4), 295-310.

Reedidbem10/ 06/ 2011

Reformuladbem28/ 08/ 2012

Aprovadbem05/ 09/ 2012

Sobre os autores:

José Maurício Haas Bueno é psicólogo, doutor pelo Programa de Pós-Graduação Strido Sensu em Psicologia da Universidade São Francisco, Itatiba/ SP, Brasil e professor no Programa de Pós-Graduação em Psicologia Cognitiva da Universidade Federal de Pernambuco, Recife/ PE, Brasil.

Lucas de Francisco Carvalho é psicólogo, doutor e professor no Programa de Pós-Graduação Strido Sensu em Psicologia da Universidade São Francisco, Itatiba/ SP, Brasil.

Ana Júlia Femandes Moreira é mestranda em Psicologia pela Universidade do Minho, Braga, Portugal.

Ana Margarida Almeida Brandão Capelo é mestranda em Psicologia pela Universidade do Minho, Braga, Portugal.

Ângela Marisa Cardoso Fernandes é mestre em Psicologia da Justiça pela Universidade do Minho, Braga, Portugal.

Margarida F. Gomes Vasconcelos é mestranda em Psicologia pela Universidade do Minho, Braga, Portugal.

Tiago André Martins Costa é mestrando em Psicologia pela Universidade do Minho, Braga, Portugal.

Vânia Sofia Leite Marques é estudante de Psicologia na Universidade do Minho, Braga, Portugal, sendo este trabalho derivado de sua atuação como Bolseira de Integração na Investigação da Fundação para a Ciência e Tecnologia - FCT.

Contato com os autores:

Centro de Filosofia e Ciências Humanas - CFCH, Curso de Psicologia. Av. Prof. Moraes Rego, 1.235 - Cidade Universitária - CEP 50670-901. Recife-PE. 